\title{
Phylogenetic Study and Divergence of Weaver Ant, Oecophylla smaragdina Fabricius in Bangladesh (Hymenoptera: Formicidae)
}

\author{
Md Mamunur Rahman ${ }^{1,2, ~}{ }^{*}$, Shingo Hosoishi ${ }^{1}$, Kazuo Ogata ${ }^{1}$ \\ ${ }^{1}$ Institute of Tropical Agriculture, Kyushu University, Motooka Nishi-ku Fukuoka, Japan \\ ${ }^{2}$ Department of Entomology, Faculty of Agriculture, Bangabandhu Sheikh Mujibur Rahman Agricultural University, Gazipur, Bangladesh
}

Email address:

mamunur111@gmail.com (Md M. Rahman)

${ }^{*}$ Corresponding author

\section{To cite this article:}

Md Mamunur Rahman, Shingo Hosoishi, Kazuo Ogata. Phylogenetic Study and Divergence of Weaver Ant, Oecophylla smaragdina Fabricius in Bangladesh (Hymenoptera: Formicidae). American Journal of Biological and Environmental Statistics.

Vol. 7, No. 3, 2021, pp. 57-66. doi: 10.11648/j.ajbes.20210703.11

Received: June 22, 2021; Accepted: July 5, 2021; Published: July 9, 2021

\begin{abstract}
The weaver ant species, Oecophylla smaragdina, is widely distributed from India through Southeast Asia to northern Australia including many tropical Western Pacific islands. Recent phylogenetic study of $O$. smaragdina showed that the species is divided into 7 groups based on mtDNA and the Bangladesh populations belong to SE Asian mainland clade despite its geographical proximity to India. The present study aims to reveal the phylogenetic aspect of $O$. smaragdina in Bangladesh with its divergence time estimation based on extensive materials. Sampling was executed according to zonation of 5 areas which are demarcated by 3 main rivers and a total of 95 colonies from 87 localities of 47 Districts were collected during 2013 to 2018 from Bangladesh. The molecular analyses, using 2 mitochondrial loci: Cytochrome b oxidase subunit 2 (Cytb) consisting of $507 \mathrm{bp}$ and Cytochrome c oxidase subunit I (COI) consisting of 639 bp done in Ku, Japan. The analysis of 84 colonies revealed that 47 are the Indian clade (Indian type) and 37 are the SE Asian mainland clade (SE Asian type). The distribution of mtDNA types showed the occurrence of both the two types with some overlapping distribution in the central part of Bangladesh. The divergence time analysis in Bangladesh populations, resulted that Indian type diverged ca. 2.2 Ma and SE Asian type diverged ca. $0.20 \mathrm{Ma}$ corresponding to early to late Pleistocene. This study suggested a comprehensive phylogenetic scenario of $O$. smaragdina in Bangladesh.
\end{abstract}

Keywords: Distribution Pattern, Divergence Time, Phylogeny, Mitochondrial DNA, Weaver Ant

\section{Introduction}

The weaver ant, $O$. smaragdina is a broadly distributed genus and has the ability to disperse over ocean islands without human interventions. Azuma et al. first analyzed populations of $O$. smaragdina using molecular data and samples, including the O. smaragdina from Bangladesh [3]. In addition, she added comprehensive samples of $O$. smaragdina from India, Southeast Asia and Australia, Azuma et al. proposed an outline of the phylogeography of $O$. smaragdina and categorized the sampled populations into 7 major clades: group 1 from India; group 2 from Southeast Asian mainland including the Indochinese and Malayan Peninsulas, as well as the Greater Sunda Islands; group 3 from the Philippines; group 4 from Flores; group 5 from Sulawesi; group 6 from Halmahera; group 7 from Australia and New Guinea [4]. Hereafter we will refer the Azuma et al. group 1 as Indian type and Azuma et al. group 2 as SE Asian type [4]. Asaka extended the survey of $O$. smaragdina to South Asia and collected several samples from India and Sri Lanka [1]. Her phylogenetic analysis showed that all analyzed samples belong to Indian clade with low levels of sequence divergence. Azuma et al. characterized the mitochondrial sequence identity of the Bangladesh populations as belonging to the Southeast Asian clade in spite of the geographical proximity of Bangladesh to India [4]. They hypothesized the existence of an Indian refuge for the independence of group 1 from group 2 and concluded that if 
the Indian population originated from the Indian refuge and Bangladesh Based on those data, Bangladesh is considered a major transition zone between Indian and Southeast Asian populations. This is the unique case of population boundaries without any distinguished geographical borders (e.g., deep sea or high mountains), although the seven groups of $O$. smaragdina based on haplotype grouping by Azuma et al. are geographically bordered by the sea [4]. In recent phylogeographic study, Rahman et al. identified the western Bangladeshi populations as Indian type and some parts of the central area has the overlapping population of both Indian and SE Asian type [22, 23]. This is the first report of occurring Indian type in Bangladesh and this evidence proved that in Bangladesh, there is no such border to restrict the occurrence of Indian type in Bangladesh. A recent study about the site records for O. smaragdina in Bangladesh has showed the same results [31]. Similar trends were also observed in the case of Asian elephants, where two highly divergent mtDNA of Asian elephants overlapped geographically due to secondary contact after glaciation during Pleistocene [30].

Oecophylla smaragdina, based on the fossil record might have originated in the early Paleogene (ca. $60 \mathrm{Ma}$ ) in the Palaearctic region, and dispersed during the climatic changes of the Eocene-Oligocene transition at ca. $43 \mathrm{Ma}$ [10]. The abrupt cooling during this period had great impact on biodiversity [14]. During this period, Earth's climate shifted from a relatively ice-free world to one with glacial conditions in polar regions characterized by substantial ice sheets [6]. Recently, Blamier et al. estimated the divergence time of the genus Oecophylla based on the fossil records and ultraconserved elements (UCEs) [5]. They estimated that Oecophylla crown group evolved during Oligocene at ca. < $30 \mathrm{Ma}$ and stem-group evolved during early Eocene at ca .50 Ma. Wetterer gave a glimpse of distribution of $O$. smaragdina in this continent with some interesting evidence of distribution [31]. Oecophylla smaragdina and $O$. longinoda have diverged in 13.3 to $11.3 \mathrm{Ma}$ ago, in the late Miocene. Diversification of seven groups occurred between the middle of Miocene to early Pliocene. While the diversification within groups was recorded between middle Pliocene to early Pleistocene [4]. There was no evidence regarding the divergence time of group 1 or Indian types. Due to widespread distribution, $O$. smaragdina was considered valuable material for biogeographic study in tropical Asia.

The purpose of the present experiment was to examine the phylogenetic relationship of weaver ant populations from northern, eastern and central parts of Bangladesh for covering its distribution range in the country and is to get insights into the estimation of the time of divergence of Indian and SE Asian types of $O$. smaragdina in Bangladesh. The previous sampling by Azuma et al. was limited to only one site of Nurbag, Gazipur, located at the central part of Bangladesh, distinguished as Southeast Asian clades [4]. However, O. smaragdina from the western parts of Bangladesh were identified as Indian clade. This result suggested the importance of phylogenetic study of central and eastern Bangladeshi populations to identify the nature of distribution with its divergence history.

\section{Materials and Methods}

\subsection{The Setting of the Study Areas and Its Characterizations}

Based on three main rivers, Ganges, Jamuna, and Meghna, the study area in Bangladesh was broadly categorized into five areas under 8 divisions. The detailed information of that 5 broad areas is presented in the following figure (Figure 1).

\section{O. Smaragdina study sites in BD}

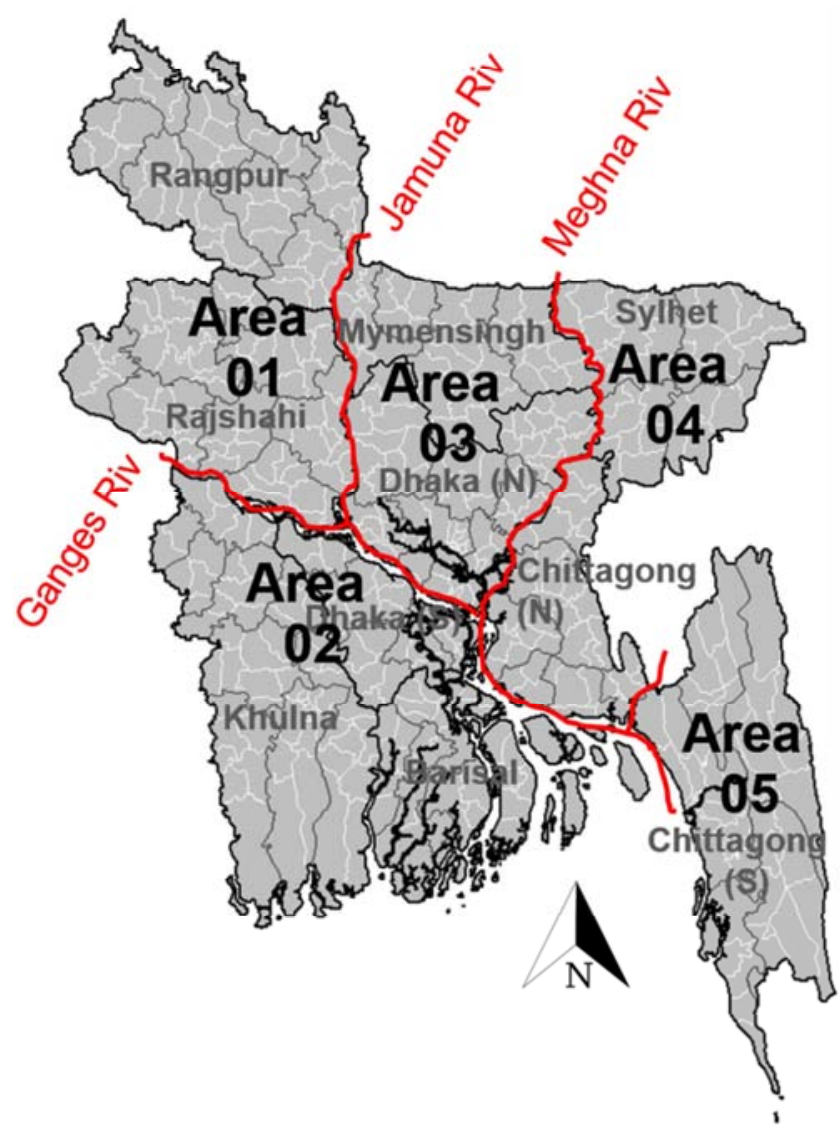

Figure 1. Sampling sites of 5 broad areas based on three main rivers in Bangladesh. The red line across the map showing the river flow that separated 5 broad areas in Bangladesh.

Area 01 included the northwestern part of Bangladesh. This area include 16 districts under 2 divisions. The climate of this area is comparatively dry with very hot summer and cold winter to other areas. This area is surrounded by the river Ganges and Jamuna. The study area lies between $24^{\circ} 07^{\prime}$ $\mathrm{N}$ to $25^{\circ} 13^{\prime} \mathrm{N}$ latitude and $88^{\circ} 00^{\prime} \mathrm{E}$ to $89^{\circ} 10^{\prime} \mathrm{E}$ longitude [32]. This northwestern part of Bangladesh has been evolved during Pleistocene Terraces due to tectonic upliftment and /or exists as an erosional geomorphic feature [24]. Area 02 was 
located at the western districts of the country consisting Khulna, Barisal and western part of Dhaka divisions. The important feature of this area is the existence of Mangrove forest, "The Sundarbans". So, this area possesses some unique vegetation. The lower boundary of that area 02 is flooded by the Bay of Bengal. The major river channels are derived from this Bay of Bengal along with the flow of Ganges. Area 03 was located in the central and northern part of the country with the northern part of Dhaka division and Mymensingh division. The major characteristics of this area is the availability of Shal forest and it is mainly covered by the plain land. This area is characterized by Pleistocene upliftment resulted Bhawal and Madhupur tract [25]. Some hilly areas including tectonically uplifted blocks created during Pleistocene are also the major characteristics in this area. This area is developed on the Ganges-Brahmaputra delta on late quaternary stratification [13]. Area 04 was located in the northern part of Chittagong division and Sylhet. Northern Chittagong is a plane landed area with plenty of fresh water sources and many rivers crisscross across while Sylhet division is mountainous with enormous number of tea garden with natural water fall. The major geological feature of that area is the Bengal basin. The Surma Basin is a subbasin of the Bengal Basin situated in the northeastern part of Bangladesh. The basin is bounded on the north by the Shillong plateau, east and southeast by the ChittagongTripura fold belt of the Indo-Burman ranges, and west by The Indian Shield platform. This area is mainly created during Holocene [18]. Area 05 includes the southern Chittagong division. This area is divided from other regions of Bangladesh by the border of Meghna rivers. It is a mountainous area and the longest sea beach, Coxsbazar, is situated in this area. The area is mainly composed of Hill Tracts (CHT) in the south-eastern part of Bangladesh encompassing three hill districts: Rangamati, Khagrachari and Bandarban. It shares borders with Myanmar on the south and southeast, India on the north and northeast, and the Chittagong district of Bangladesh on the west. CHT is located between $21^{\circ} 40^{\prime}$ degrees and $23^{\circ} 47^{\prime}$ degrees north latitude and $91^{\circ} 40^{\prime}$ degrees and $92^{\circ} 42^{\prime}$ degrees east longitude.

\subsection{Localities of O. smaragdina Sampling in Bangladesh}

A total of $95 O$. smaragdina colonies had been collected from 87 localities of 47 districts belonging to 8 divisions of Bangladesh during September 2013 to December 2018. The locality position and detailed information are presented in Figure 2 and Table 1.

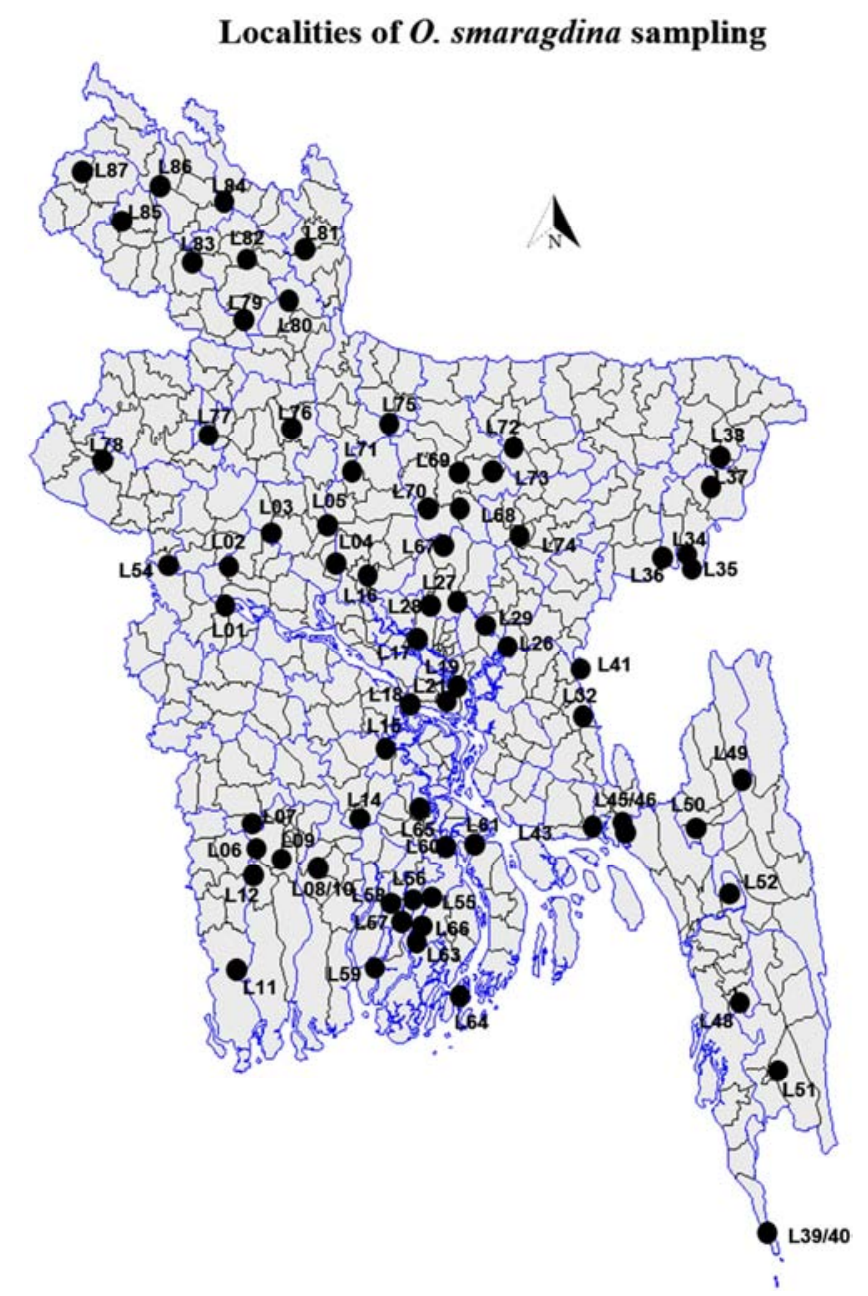

Figure 2. Locality based sampling sites of Oecophylla smaragdina in Bangladesh.

Table 1. Detailed locality information of O. smaragdina sampling sites in Bangladesh.

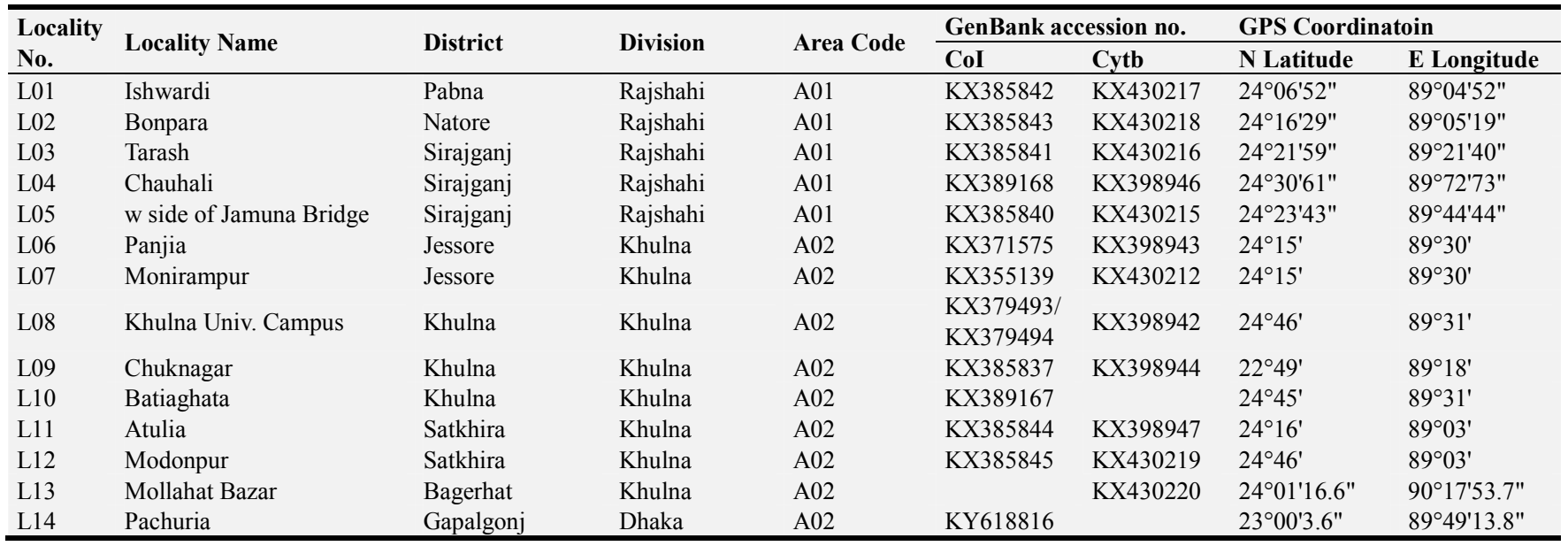




\begin{tabular}{|c|c|c|c|c|c|c|c|c|}
\hline \multirow{2}{*}{$\begin{array}{l}\text { Locality } \\
\text { No. }\end{array}$} & \multirow{2}{*}{ Locality Name } & \multirow{2}{*}{ District } & \multirow{2}{*}{ Division } & \multirow{2}{*}{ Area Code } & \multicolumn{2}{|c|}{ GenBank accession no. } & \multicolumn{2}{|c|}{ GPS Coordinatoin } \\
\hline & & & & & CoI & Cytb & N Latitude & E Longitude \\
\hline $\mathrm{L} 15$ & Bhanga & Faridpur & Dhaka & A02 & KX389172 & & $23^{\circ} 23^{\prime} 0^{\prime \prime}$ & $89^{\circ} 59^{\prime} 0^{\prime \prime}$ \\
\hline L16 & Elenga & Tangail & Dhaka & A03 & KX385839 & KX398945 & $24^{\circ} 23^{\prime} 01^{\prime \prime}$ & $89^{\circ} 50^{\prime} 05^{\prime \prime}$ \\
\hline L17 & Kumrail & Dhaka & Dhaka & A03 & KX389169 & & $23^{\circ} 13 ' 26.1^{\prime \prime}$ & $90^{\circ} 13^{\prime} 26.1^{\prime \prime}$ \\
\hline L18 & Thanamore & Dhaka & Dhaka & A03 & KX389170 & & $23^{\circ} 36^{\prime} 49.4^{\prime \prime}$ & $90^{\circ} 07^{\prime} 13.3^{\prime \prime}$ \\
\hline L19 & Ruhitpur & Dhaka & Dhaka & A03 & & KY562567 & $23^{\circ} 40^{\prime} 07^{\prime \prime}$ & $90^{\circ} 17^{\prime} 54^{\prime \prime}$ \\
\hline L20 & Baluakandi & Munshiganj & Dhaka & A03 & MG967475 & & $23^{\circ} 38^{\prime} 57.2^{\prime \prime}$ & $90^{\circ} 35^{\prime} 8.2^{\prime \prime}$ \\
\hline L21 & Nimtali & Munshiganj & Dhaka & $\mathrm{A} 03$ & KY628426 & & $23^{\circ} 36^{\prime} 55.3^{\prime \prime}$ & $90^{\circ} 19^{\prime} 55.6^{\prime \prime}$ \\
\hline L22 & Bejgaon & Munshiganj & Dhaka & A03 & & KY583087 & $23^{\circ} 32^{\prime} 21.3^{\prime \prime}$ & $90^{\circ} 17^{\prime} 48.2^{\prime \prime}$ \\
\hline L23 & Nababganj Bazar & Narayanganj & Dhaka & $\mathrm{A} 03$ & & MG967471 & $23^{\circ} 39^{\prime} 39.2^{\prime \prime}$ & $90^{\circ} 09^{\prime} 41.9^{\prime \prime}$ \\
\hline L24 & Shiddirganj & Narayanganj & Dhaka & A03 & & KY562571 & $23^{\circ} 41^{\prime} 41.8^{\prime \prime}$ & $90^{\circ} 29^{\prime} 26.5^{\prime \prime}$ \\
\hline L25 & Vulta & Narayanganji & Dhaka & A03 & & KY562573 & $23^{\circ} 46^{\prime} 50^{\prime \prime}$ & $90^{\circ} 34^{\prime} 16.5^{\prime \prime}$ \\
\hline L26 & Panchdona & Norsingdi & Dhaka & A03 & KY657490 & KY562569 & $23^{\circ} 53^{\prime} 36.2^{\prime \prime}$ & $90^{\circ} 34^{\prime} 16.5^{\prime \prime}$ \\
\hline L27 & Bhawal National Park & Gazipur & Dhaka & A03 & KX385838 & KX430214 & $24^{\circ} 04^{\prime} 43^{\prime \prime}$ & $90^{\circ} 24^{\prime} 06^{\prime \prime}$ \\
\hline L28 & Nurbag & Gazipur & Dhaka & A03 & KX389171 & KX430221 & $24^{\circ} 01^{\prime} 16.6^{\prime \prime}$ & $90^{\circ} 17^{\prime} 53.7^{\prime \prime}$ \\
\hline L29 & Charpara & Gazipur & Dhaka & A03 & KY628425 & & $23^{\circ} 55^{\prime} 31.9^{\prime \prime}$ & $90^{\circ} 36^{\prime} 0.8^{\prime \prime}$ \\
\hline L30 & Zamirarchala & Gazipur & Dhaka & A03 & & & $24^{\circ} 06^{\prime} 6.2^{\prime \prime}$ & $90^{\circ} 28^{\prime} 25.8^{\prime \prime}$ \\
\hline L31 & Rajbari & Gazipur & Dhaka & A03 & & KY562568 & $24^{\circ} 03^{\prime} 5.7^{\prime \prime}$ & $90^{\circ} 23^{\prime} 17.8^{\prime \prime}$ \\
\hline L32 & Nimshar & Comilla & Chittagong & A05 & MG967473 & MG967472 & $23^{\circ} 06^{\prime} 6.2^{\prime \prime}$ & $90^{\circ} 28^{\prime} 25.8^{\prime \prime}$ \\
\hline L33 & Madhoyia & Comilla & Chittagong & $\mathrm{A} 05$ & & & $23^{\circ} 30^{\prime} 08.5^{\prime \prime}$ & $91^{\circ} 0^{\prime} 30^{\prime \prime}$ \\
\hline L34 & Tea Resort Center & Moulovibazar & Sylhet & A04 & KY618809 & KY583084 & $24^{\circ} 18^{\prime} 7.2^{\prime \prime}$ & $91^{\circ} 45^{\prime} 34.92$ \\
\hline L35 & Lauachra National Park & Moulovibazar & Sylhet & A04 & KY618815 & KY583085 & $24^{\circ} 18^{\prime} 05.7^{\prime \prime}$ & $91^{\circ} 45^{\prime} 41.0^{\prime \prime}$ \\
\hline L36 & Bahubal & Habiganj & Sylhet & A04 & KY618818 & KY583083 & $24^{\circ} 22^{\prime} 49^{\prime \prime}$ & $91^{\circ} 24^{\prime} 46.0^{\prime \prime}$ \\
\hline L37 & Tarau & Sylhet & Sylhet & A04 & KY618817 & & $24^{\circ} 19^{\prime} 42.6^{\prime \prime}$ & $91^{\circ} 47^{\prime} 05.2^{\prime \prime}$ \\
\hline L38 & Doradarpur & Sylhet & Sylhet & A04 & KY618810 & KY657484 & $24^{\circ} 46^{\prime} 40.0^{\prime \prime}$ & $91^{\circ} 47^{\prime} 01.8^{\prime \prime}$ \\
\hline L39 & Marishbunia & Cox's Bazar & Chittagong & A05 & MG967474 & & $21^{\circ} 05^{\prime} 25^{\prime \prime}$ & $92^{\circ} 20^{\prime}$ \\
\hline L40 & Noakhali para & Cox's Bazar & Chittagong & A05 & & & $24^{\circ} 46^{\prime} 40.0^{\prime \prime}$ & $91^{\circ} 47^{\prime} 01.8^{\prime \prime}$ \\
\hline L41 & Mondabag & Bramhanbaria & Chittagong & A04 & KY608802 & KY657492 & $23^{\circ} 44^{\prime} 0^{\prime \prime}$ & $91^{\circ} 10^{\prime} 0^{\prime \prime}$ \\
\hline L42 & Jogotpur & Comilla & Chittagong & A04 & & & $23^{\circ} 33^{\prime} 0^{\prime \prime}$ & $91^{\circ} 7^{\prime} 36^{\prime \prime}$ \\
\hline L43 & Sebarhat & Noakhali & Chittagong & A04 & KY608803 & KY550396 & $22^{\circ} 58^{\prime} 59.88^{\prime \prime}$ & $91^{\circ} 13^{\prime} 59.88^{\prime \prime}$ \\
\hline L44 & Senbag Upozilla Hospital & Noakhali & Chittagong & A04 & & KY657493 & $22^{\circ} 58^{\prime} 59.18^{\prime \prime}$ & $91^{\circ} 13^{\prime} 59.08^{\prime \prime}$ \\
\hline L45 & Mohipal & Feni & Chittagong & A04 & KY618811 & KY550397 & $23^{\circ} 1^{\prime} 0.89^{\prime \prime}$ & $91^{\circ} 23^{\prime} 30^{\prime \prime}$ \\
\hline L46 & Mohipal Primary School & Feni & Chittagong & A04 & KY628427 & KY550398 & $23^{\circ} 1^{\prime} 1.10^{\prime \prime}$ & $91^{\circ} 23^{\prime} 50^{\prime \prime}$ \\
\hline L47 & Raujan Bazar & Chittagong & Chittagong & A05 & & KY550399 & $22^{\circ} 32^{\prime} 0^{\prime \prime}$ & $91^{\circ} 56^{\prime} 0^{\prime \prime}$ \\
\hline L48 & Satkania & Chittagong & Chittagong & A05 & KY657489 & KY550400 & $22^{\circ} 6^{\prime} 12^{\prime \prime}$ & $92^{\circ} 4^{\prime} 50^{\prime \prime}$ \\
\hline L49 & Dighinala HRC & Khagrachari & Chittagong & A05 & KY608804 & KY550401 & $23^{\circ} 15^{\prime} 30^{\prime \prime}$ & $92^{\circ} 3^{\prime} 30^{\prime \prime}$ \\
\hline L50 & Matiranga Dhibi & Khagrachari & Chittagong & A05 & KY608805 & KY550402 & $23^{\circ} 2^{\prime} 30.12^{\prime \prime}$ & $91^{\circ} 52^{\prime} 30^{\prime \prime}$ \\
\hline L51 & Ruma Karai & Bandarban & Chittagong & A05 & KY657488 & KY550403 & $22^{\circ} 3^{\prime} 0^{\prime \prime}$ & $92^{\circ} 25^{\prime} 0.12^{\prime \prime}$ \\
\hline L52 & Kawkhali Bazar & Rangamati & Chittagong & A05 & KY583089 & & $22^{\circ} 32^{\prime} 0^{\prime \prime}$ & $92^{\circ} 1^{\prime} 0^{\prime \prime}$ \\
\hline L53 & Patia & Chittagong & Chittagong & $\mathrm{A} 05$ & & KY562572 & $22^{\circ} 18^{\prime} 0^{\prime \prime}$ & $91^{\circ} 59^{\prime} 0^{\prime \prime}$ \\
\hline L54 & Thanapara Sadah & Rajshahi & Rajshahi & A01 & KY628429 & & $24^{\circ} 17^{\prime} 0^{\prime \prime}$ & $88^{\circ} 46^{\prime} 30^{\prime \prime}$ \\
\hline L55 & Nalchiti prim. sch. field & Jhalokati & Barisal & $\mathrm{A} 02$ & KY618814 & KY657585 & $22^{\circ} 37^{\prime} 19.92^{\prime \prime}$ & $90^{\circ} 16^{\prime} 14.88^{\prime \prime}$ \\
\hline L56 & BRAC more & Jhalokati & Barisal & $\mathrm{A} 02$ & KY657491 & KY550389 & $22^{\circ} 38^{\prime} 36^{\prime \prime}$ & $90^{\circ} 12^{\prime} 0^{\prime \prime}$ \\
\hline L57 & Baghribazar & Jhalokati & Barisal & $\mathrm{A} 02$ & KY618813 & KY550388 & $22^{\circ} 40^{\prime} 0.12^{\prime \prime}$ & $90^{\circ} 8^{\prime} 30.12^{\prime \prime}$ \\
\hline L58 & Kawkhali Upz P Chottor & Pirojpur & Barisal & $\mathrm{A} 02$ & KY583090 & KY550390 & $22^{\circ} 37^{\prime} 13.08^{\prime \prime}$ & $90^{\circ} 4^{\prime} 9.84^{\prime \prime}$ \\
\hline L59 & Shakharikathi & Pirojpur & Barisal & $\mathrm{A} 02$ & KY583091 & KY550391 & $22^{\circ} 17^{\prime} 12.84^{\prime \prime}$ & $89^{\circ} 58^{\prime} 0.12^{\prime \prime}$ \\
\hline L60 & Rupatoli & Barisal & Barisal & $\mathrm{A} 02$ & & KY550395 & $22^{\circ} 48^{\prime} 0^{\prime \prime}$ & $90^{\circ} 30^{\prime} 0^{\prime \prime}$ \\
\hline L61 & Patarhat & Barisal & Barisal & $\mathrm{A} 02$ & KY583092 & KY550392 & $22^{\circ} 49^{\prime} 54.84^{\prime \prime}$ & $90^{\circ} 31^{\prime} 59.88^{\prime \prime}$ \\
\hline L62 & Barguna Sadar bus stand & Barguna & Barisal & $\mathrm{A} 02$ & & KY550393 & $22^{\circ} 9^{\prime} 2.88^{\prime \prime}$ & $90^{\circ} 7^{\prime} 35.04^{\prime \prime}$ \\
\hline L63 & PSTU & Patuakhali & Barisal & A02 & & KY550394 & $22^{\circ} 26^{\prime} 0^{\prime \prime}$ & $90^{\circ} 22^{\prime} 0^{\prime \prime}$ \\
\hline L64 & Panpatti & Patuakhali & Barisal & $\mathrm{A} 02$ & KY583093 & KY583088 & $22^{\circ} 9^{\prime} 48^{\prime \prime}$ & $90^{\circ} 25^{\prime} 48^{\prime \prime}$ \\
\hline L65 & Agailjhara Uni P office & Barisal & Barisal & $\mathrm{A} 02$ & KY628430 & & $22^{\circ} 58^{\prime} 0^{\prime \prime}$ & $90^{\circ} 9^{\prime} 0^{\prime \prime}$ \\
\hline L66 & Mohespur & Barisal & Barisal & $\mathrm{A} 02$ & KY618812 & KY657486 & $22^{\circ} 33^{\prime} 0^{\prime \prime}$ & $90^{\circ} 20^{\prime} 18^{\prime \prime}$ \\
\hline L67 & Bhaluka Bazar & Mymensingh & Mymensingh & A03 & KY657499 & & $24^{\circ} 22^{\prime} 30^{\prime \prime}$ & $90^{\circ} 22^{\prime} 42^{\prime \prime}$ \\
\hline L68 & Trishal Primary School & Mymensingh & Mymensingh & $\mathrm{A} 03$ & KY657500 & & $24^{\circ} 34^{\prime} 30^{\prime \prime}$ & $90^{\circ} 23^{\prime} 30^{\prime \prime}$ \\
\hline L69 & BAU Campus & Mymensingh & Mymensingh & A03 & KY657501 & & $24^{\circ} 45^{\prime} 8.39^{\prime \prime}$ & $90^{\circ} 24^{\prime} 6.59^{\prime \prime}$ \\
\hline L69 & BAU, Sesh Matha More & Mymensingh & Mymensingh & A03 & KY657502 & & $24^{\circ} 45^{\prime} 8.39^{\prime \prime}$ & $90^{\circ} 24^{\prime} 6.59^{\prime \prime}$ \\
\hline L70 & Nandail & Mymensingh & Mymensingh & A03 & KY657503 & KY657505 & $24^{\circ} 34^{\prime} 0^{\prime \prime}$ & $90^{\circ} 41^{\prime} 0^{\prime \prime}$ \\
\hline L71 & Sarisha Bari High School & Jamalpulr & Mymensingh & A03 & KY657504 & KY657506 & $24^{\circ} 44^{\prime} 30^{\prime \prime}$ & $89^{\circ} 50^{\prime} 0^{\prime \prime}$ \\
\hline L71 & Bazar Pukur & Jamalpur & Mymensingh & A03 & & KY65707 & $24^{\circ} 44^{\prime} 32^{\prime \prime}$ & $89^{\circ} 50^{\prime} 01^{\prime \prime}$ \\
\hline L72 & Sadar Hospital & Netrokona & Mymensingh & A03 & KY657494 & & $24^{\circ} 52^{\prime} 30^{\prime \prime}$ & $90^{\circ} 44^{\prime} 0^{\prime \prime}$ \\
\hline L72 & Sadar Primary School & Netrokona & Mymensingh & A03 & KY657495 & & $24^{\circ} 52^{\prime} 28^{\prime \prime}$ & $90^{\circ} 44^{\prime} 03^{\prime \prime}$ \\
\hline L73 & Gauripur Upz Complex & Mymensingh & Mymensingh & A03 & KY657496 & & $24^{\circ} 45^{\prime} 30^{\prime \prime}$ & $90^{\circ} 34^{\prime} 30^{\prime \prime}$ \\
\hline L74 & Higher par & Kishorgonj & Mymensingh & A03 & KY657497 & & $24^{\circ} 25^{\prime} 59.88$ & $90^{\circ} 46^{\prime} 59.88^{\prime \prime}$ \\
\hline L75 & Sadar Thana more & Sherpur & Mymensingh & A03 & KY657498 & & $25^{\circ} 0^{\prime} 0^{\prime \prime}$ & $90^{\circ} 1^{\prime} 0^{\prime \prime}$ \\
\hline L76 & Dhunat Upz. Chattor & Bogra & Rajshahi & A01 & MG873538 & MG886852 & $24^{\circ} 41^{\prime} 10.00^{\prime \prime}$ & $89^{\circ} 32^{\prime} 0.03^{\prime \prime}$ \\
\hline L77 & Municipality orchard & Naogaon & Rajshahi & $\mathrm{A} 01$ & MG873539 & MG886851 & $24^{\circ} 48^{\prime} 18.00^{\prime \prime}$ & $88^{\circ} 57^{\prime} 0.18^{\prime \prime}$ \\
\hline
\end{tabular}




\begin{tabular}{|c|c|c|c|c|c|c|c|c|}
\hline \multirow{2}{*}{$\begin{array}{l}\text { Locality } \\
\text { No. }\end{array}$} & \multirow{2}{*}{ Locality Name } & \multirow{2}{*}{ District } & \multirow{2}{*}{ Division } & \multirow{2}{*}{ Area Code } & \multicolumn{2}{|c|}{ GenBank accession no. } & \multicolumn{2}{|c|}{ GPS Coordinatoin } \\
\hline & & & & & CoI & Cytb & N Latitude & E Longitude \\
\hline $\mathrm{L} 78$ & Nijampur & Chapainawabganj & Rajshahi & A01 & MG873540 & MG886850 & $24^{\circ} 43^{\prime} 48.03^{\prime \prime}$ & $88^{\circ} 25^{\prime} 12.13^{\prime \prime}$ \\
\hline L79 & Hakimpur Nursery & Dinajpur & Rangpur & A01 & MG873541 & MG886847 & $25^{\circ} 16^{\prime} 59.88^{\prime \prime}$ & $89^{\circ} 1^{\prime} 0.12^{\prime \prime}$ \\
\hline L80 & Shibpur & Gaibandha & Rangpur & A01 & MG873542 & MG886849 & $25^{\circ} 07^{\prime} 59.88^{\prime \prime}$ & $89^{\circ} 23^{\prime} 30.12^{\prime \prime}$ \\
\hline L81 & Gharialdanga & Kurigram & Rangpur & A01 & MG873543 & & $25^{\circ} 48^{\prime} 00^{\prime \prime}$ & $89^{\circ} 33^{\prime} 0.02^{\prime \prime}$ \\
\hline L82 & Rasulpur School ground & Rangpur & Rangpur & A01 & MG873544 & MG886845 & $25^{\circ} 24^{\prime} 54^{\prime \prime}$ & $89^{\circ} 19^{\prime} 0.10^{\prime \prime}$ \\
\hline L83 & Saidpur airport surrounding & Nilfamari & Rangpur & $\mathrm{A} 01$ & MG873545 & & $25^{\circ} 47^{\prime} 0.04^{\prime \prime}$ & $88^{\circ} 54^{\prime} 0.02^{\prime \prime}$ \\
\hline L85 & Pirganj fire station orchard & Thakurgaon & Rangpur & A01 & MG873547 & MG886848 & $25^{\circ} 51^{\prime} 15.12^{\prime \prime}$ & $88^{\circ} 22^{\prime} 0.12^{\prime \prime}$ \\
\hline L86 & Debiganj bus stand & Panchagar & Rangpur & $\mathrm{A} 01$ & MG873548 & MG886844 & $26^{\circ} 07^{\prime} 9.89^{\prime \prime}$ & $88^{\circ} 45^{\prime} 33.47^{\prime \prime}$ \\
\hline L87 & Atwari sadar thana more & Panchagar & Rangpur & A01 & MG873549 & & $26^{\circ} 18^{\prime} 29.88^{\prime \prime}$ & $88^{\circ} 27^{\prime} 29.88^{\prime \prime}$ \\
\hline
\end{tabular}

\subsection{Sample Preparation}

The collected samples from Bangladesh were analyzed for sequencing in the laboratory Institute of Tropical Agriculture of Kyushu University, Japan. The collected colonies of Oecophylla smaragdina were preserved in $99 \%$ ethanol prior to DNA extraction.

\subsection{Molecular Study}

Genomic DNA was extracted from the legs of specimens that were preserved in alcohol by using QIAGEN DNeasy Blood and Tissue kit (Qiagen, Maryland, USA). Amplification of both mitochondrial and nuclear DNA was done by polymerase chain reaction (PCR). The primers used for amplification are identical to primers reported by Crozier et al., Lunt et al., Azuma et al., and Azuma et al. [9, 17, 3, 4]. For, mitochondrial DNA analysis, Primers for the Cytb gene fragment were

$\mathrm{Cb} 1$ (5'TATGTACTACCATGAGGACAAATATC' 3 ) and tRs (5'TATTTCTTTATTATGTTTTCAAAAC'3). For the COI gene fragment, COI 1 3(5'ATAATTTTTTTTATAGTTATACC'3) and COI 2-4 (5'TCCTAAAAAATGTTGAGGAAA'3) were used as forward and reverse primers, respectively by Crozier and Crozier [8]. The thermal cycling parameters for Cytb and COI basically followed the protocols established by Crozier and Crozier and Sameshima et al., including $95^{\circ} \mathrm{C}$ for $5 \mathrm{~min}$ for initial denaturation, 35 cycles of dissociation $\left(92^{\circ} \mathrm{C}, 1\right.$ min), annealing $\left(50^{\circ} \mathrm{C}\right.$ for Cytb and $54^{\circ} \mathrm{C}$ for COI, $\left.1 \mathrm{~min}\right)$, and extension $\left(70^{\circ} \mathrm{C}, 2 \mathrm{~min}\right)[8,28]$. Illustra ExoProStar was followed according to the instruction of the manufacturer GE Healthcare. For cycle sequencing, ABI PRISM Big Dye Terminator $\mathrm{v} 3.1$ cycle sequencing kits from Applied Biosystems were used in an automated sequencer. Sequencing reactions were performed by using ABI 3100 Avant DNA Sequencer (Applied Biosystems).

\subsection{Phylogenetic and Divergence Tree Inference}

For the phylogenetic analysis of $O$. smaragdina populations, combination of mitochondrial cytochorome $\mathrm{b}$ and cytochorome oxidase subunit-1 gene of $1143 \mathrm{bp}$ were used in the analysis. Of which, 63 samples for Cytb and 72 samples for COI genes have been used with 504 bp and 639 bp, respectively. In addition, sequence data of both COI and Cytb were used from Azuma et al., Azuma et al. and Asaka as reference [3, 4, 1]. The sequence data of both COI and Cytb of Oecophylla longinoda from Cameroon were used as outgroup in this analysis. The sequencing alignment was done by using Vector NTI Advance ver. 11.5 software. The sequences of Cytb and COI were aligned by using MEGA 6.0 software [29]. Phylogenetic trees were inferred from 106 concatenated matrix sequences of both COI and Cytb genes, conducted by MrBayes 3.1.2 with 1,000,000 generations $[19,27,12]$. For the selection of best- fit model, MrModeltest 2.3 was performed with PAUP*4.0b10. [21, 20]. The substitution model, GTR + I + G was used in mitochondrial $\mathrm{COI}$ and Cytb genes were used the alignment was partitioned into 1st, 2nd and 3rd nucleotide positions. The nucleotide sequences for both Cytb and COI were deposited in the GenBank with accession number are corresponding to Table 1 . For estimating the divergence time of $O$. smaragdina in Bangladesh, a total of seven localities nucleotide sequence data of Cytochrome oxidase subunit 1 (COI) and Cytb data were used. Those seven localities include L07, L08, L26, L32, L41, L49, L66, respectively. The detailed locality information are presented in Table 1. Like the phylogeny analysis, in this case, sequence data of both COI and Cytb were used from Azuma et al., Azuma et al. and Asaka retrieved from DDBJ GenBank with the samples from Malaysian and Cameroon as references [3, 4, 1]. The sequences of Oecophylla longinoda from Cameroon were used as outgroup in this analysis. The sequencing analysis was done by using Vector NTI Advance ver. 11.5 software. Haplotypes of Cytb and COI were aligned by using MEGA 6.0 software [29]. A total of 1149 bp of COI and Cytb nucleotide sequences of the samples collected from those 7 localities were used along. A Bayesian Markov chain Monte Carlo (MCMC) packages [11], which relies on a relaxed molecular clock approach, were used to estimate the divergence time. Optimal nucleotide substitutional model were chosen by Mr. ModelTest.

\section{Results}

\subsection{Bayesian Phylogenetic Tree Inferences}

Among identified 197 variable characters, 133 were parsimony informative. The Bayesian analysis of the mitochondrial concatenated matrix dataset of $1143 \mathrm{bp}$ showed that the Bangladeshi $O$. smaragdina samples were nested into two distinct clades (posterior probability $>90 \%$ ) (Figure 3). Bangladeshi 48 weaver ant samples were nested with Indian clade of $O$. smaragdina, whereas 39 samples were nested with the Southeast Asian clades. Therefore, the occurrence of both 
the Indian and SE Asian types was found within Bangladesh. Based on the phylogenetic tree obtained, the Bangladeshi populations showed the overlapping distribution of the Indian and SE Asian types of $O$. smaragdina (Figure 3).

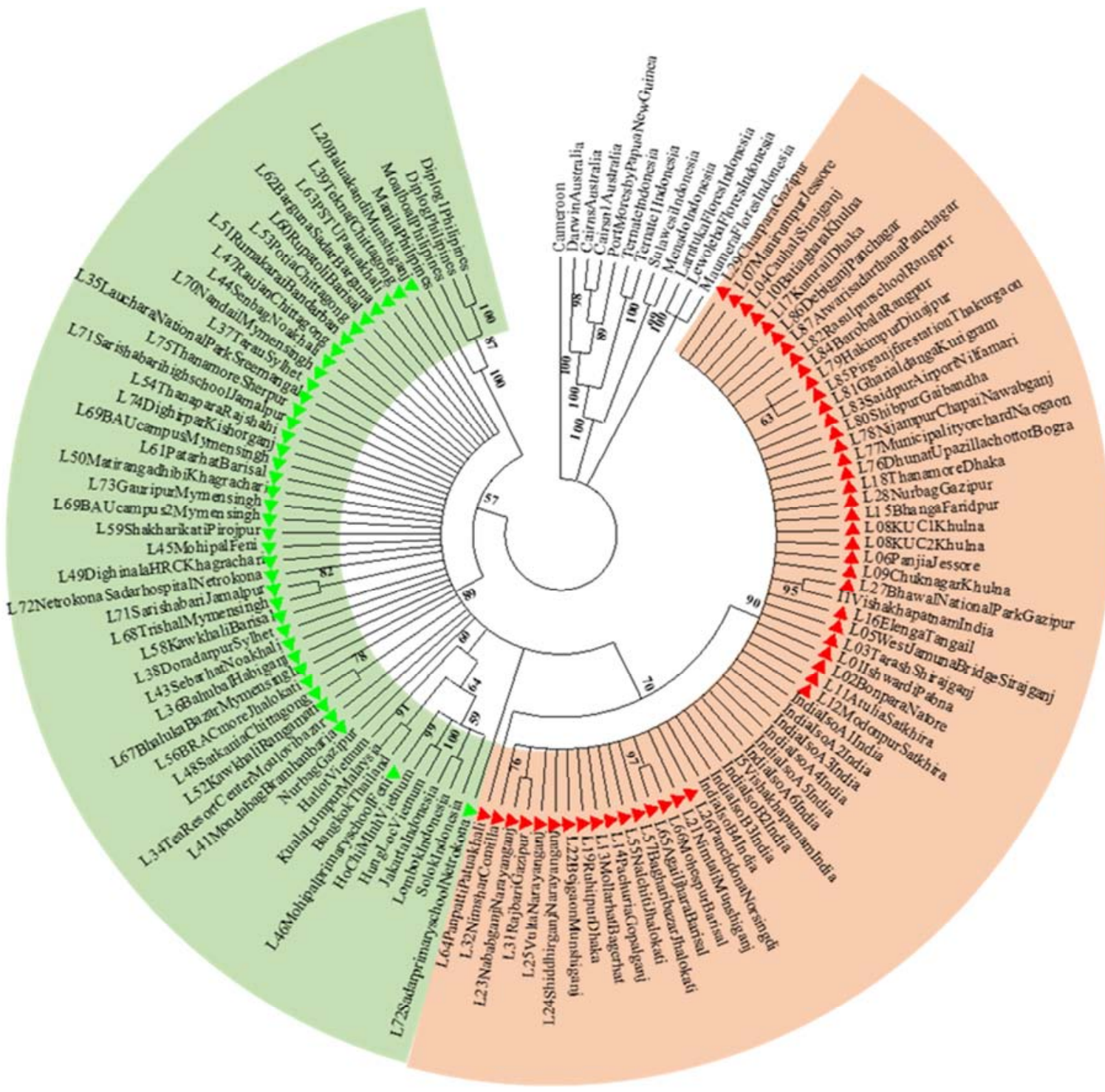

Figure 3. Bayesian phylogenetic tree of Bangladeshi O. smaragdina populations as inferred from the mitochondrial gene fragments (1143bp) of the COI (639 bp) and the Cytb $(504 \mathrm{bp})$ genes The number ahead of each locality indicates the locality number. Red and green triangle denotes the samples from Bangladesh clustering with Indian and SE Asian types, respectively.

\subsection{Summary of the Distribution Pattern}

The western part of Bangladesh was mainly occupied by Indian types, the Eastern part was dominated by SE Asian types, while, the mixture of two clades was found in the central parts of Bangladesh (Figure 4). The detailed samples results based on type are showed in Table 2. Out of 87 localities, we failed to detect 3 locality types of Oecophylla. The samples from rest 47 localities were identified as Indian type and 37 locality samples as SE Asian types (Table 2).

Table 2. Detailed summary of the distribution pattern of weaver ant in Bangladesh.

\begin{tabular}{|c|c|c|c|c|c|c|}
\hline \multirow{2}{*}{ Sampled area } & \multirow{2}{*}{ Surveyed district } & \multirow{2}{*}{ Sampled colonies } & \multirow{2}{*}{ Sampled localities } & \multicolumn{2}{|c|}{ Type (in locality) } & \multirow{2}{*}{ Failed } \\
\hline & & & & Indian & SE Asian & \\
\hline Area 01 & 14 & 18 & 18 & 17 & 1 & \\
\hline Area 02 & 11 & 23 & 22 & 15 & 7 & \\
\hline Area 03 & 10 & 30 & 25 & 13 & 11 & 1 \\
\hline Area 04 & 7 & 15 & 13 & 2 & 10 & 1 \\
\hline Area 05 & 5 & 9 & 9 & 0 & 8 & 1 \\
\hline Total & 47 & 95 & 87 & 47 & 37 & 3 \\
\hline
\end{tabular}




\section{Distribution pattern inferred by mtDNA}

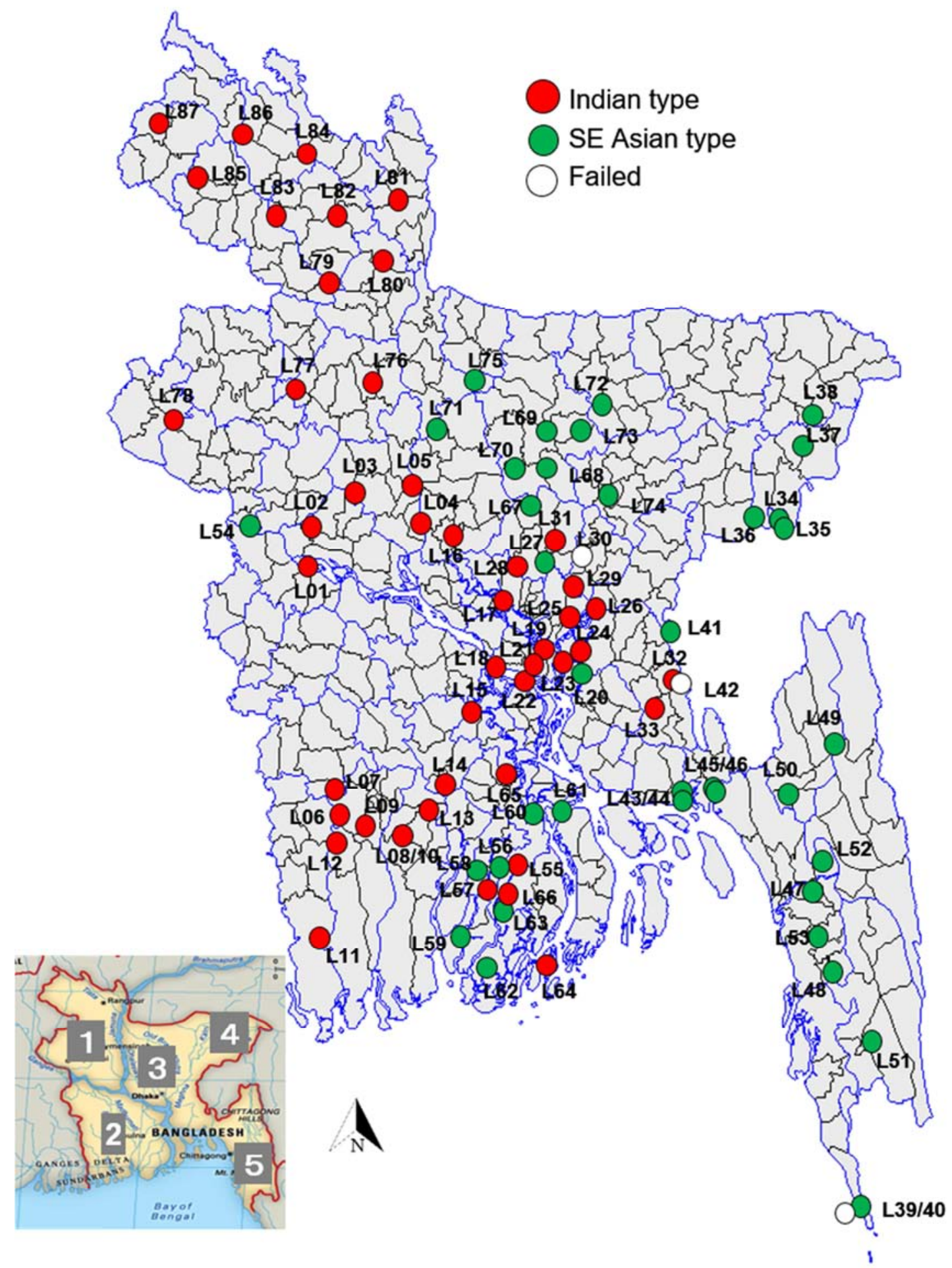

Figure 4. Distribution pattern of Indian and SE Asian types of O. smaragdina in Bangladesh.

\subsection{Divergence Time of O. smaragdina in Bangladesh}

The results of divergence time are shown in Figure 5 in the MCMC divergence tree. In this tree, sequences of the haplotypes from Cameroon are considered as out group, and
Malaysian and Indian haplotypes were used as reference. Although these divergence tree is not showing quite a good resolution due to lack of so many resolved sister groups but we can get some interesting evidence. According to this divergence tree, Indian type diverged ca. 2.2 Ma and SE 
Asian type diverged ca. 0.20 Ma corresponding to early to late Pleistocene.

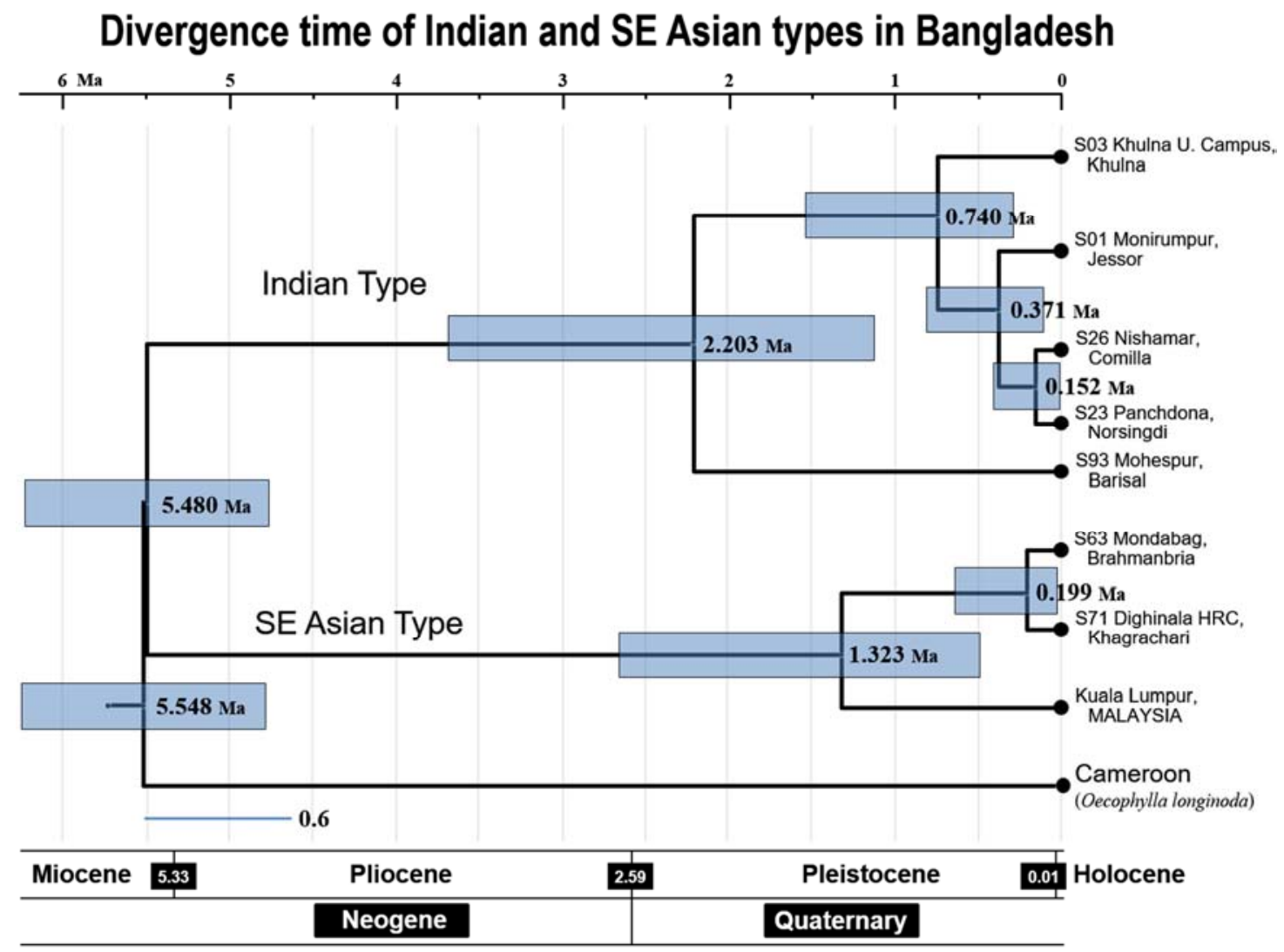

Figure 5. Divergence time of Indian and SE Asian types of O. smaragdina in Bangladesh.

\section{Discussions}

The result of distribution of $O$. smaragdina based on mtDNA showed that the western parts of Bangladesh are predominantly occupied by the Indian type, whereas the eastern part by the SE Asian type, and that the central parts is the mixture of both types. The study does not show the clear boundary, but suggests the central part as a transition zone. Divergence time of the ant genus Oecophylla is thought to be a significant factor of such distribution. Diversification within groups in this continent was recorded from the Middle Pliocene to Early Pleistocene [3]. After this period, the world has encountered a significant climatic change. It might also affect the distribution of Oecophylla in different parts of the world. Lokkers suggested two limiting factors, low temperature and humidity for distribution range of Oecophylla in Australia [15]. From this tree, we get the information regarding the divergence time of the Indian type that was missing in the previous study by Azuma et al. [3]. The quaternary paleo-geographic history of the SE Asia during the ice age showed some significant evidence of the divergence pattern. Vidya et al. discussed the distribution of the two clades of Asian elephants and proved the effect of allopatrically in different glacial refugia, the alpha clade in the Myanmar region and the beta clade possibly in southern India-Sri Lanka, 1.6-2.1 Ma ago [30]. Results from nested clade and dispersal-vicariance analyses indicate a subsequent isolation and independent diversification of the $\mathrm{b}$ clade in both Sri Lanka and the Sunda region, followed by northward expansion of the clade. Pleistocene glaciation has significant influential effects on temperature and rainfall that favored the diversification of several ant species including Oecophylla distribution [15]. Rainfall moderate the vegetation density while low temperature inhibiting larval development. As Pleistocene glaciation had the effect on both these two limiting factors of $O$. smaragdina distribution so the divergence resulted [15]. According to our study, diversification of both the types occurred in Pleistocene. There is a rapid fluctuation of temperature from late Pliocene to Pleistocene. It might influenced the distribution of Oecophylla in different part of the world. During that time, fragmentation and expansion of Oecophylla population happened. During LGM, the tropic changed from North to southward and it retained to north after glaciation. Thus, Bangladesh become vacant after glaciation with the tropical climate and hence the occurrence of the both the types have been found subsequently. Effects of temperature and rainfall influence the distribution of $O$. smaragdina as the combination of high temperature and high rainfall is necessary to continue the weaver ant growth rate and distribution, and to a limited extent, higher temperatures can compensate for lower than optimal rainfall levels, and vice versa [15]. During the glaciation period the temperature had fallen down nearly $10^{\circ} \mathrm{C}$ in Vostok might have significant 
influence on the distribution of several species like Oecophylla [16], which is markedly affected by low temperature for their colony development. Due to several glaciation during Pleistocene could be a key factor of such effects. Weterrer showed a comprehensive distribution pattern of $O$. smaragdina in the arid, tropical and subtropical climatic region that also shed light on the effects of temperature on the distribution [31].

The occurrence of two types (Indian type and SE Asian type) in Bangladesh will be made by the following dispersal scenario: The disjunction of populations would occur several times as in shown of divergent time in 7 major groups of mtDNA, and the isolated populations may develop their unique haplotypes in India, in SE Asia, or others during the history of $O$. smaragdina which was estimated to diverged from longinoda about 13 - $11 \mathrm{Ma}$ [4, 5]. Before the Last Glacial Maximum (LGM: 25,000 - 15,000 BP) the haplotypes observed in Bangladesh would be already established. During the LGM, the Bengal Delta was supposed to be vacant for Oecophylla smaragdina distribution because the northern limit of the species would be located down to the south. After the LGM, the populations expanded to north, and because of the geographical shape the east and west populations met in the Bengal Delta. When the two types of population groups met again in the Bengal Delta, each of the types already contained several haplotypes of $m t \mathrm{DNA}$. This vacant-reunion hypothesis requires a temperature drop in LGM, and diversification of populations before LGM. The present distribution of Oecophylla smaragdina, in particular, the northern limit of distribution almost agrees with the isothermal line of $10^{\circ} \mathrm{C}$ in the average temperature of January. Braconnot et al. estimated the mean temperature of the coldest month (MTCO) in Asia is around $10^{\circ} \mathrm{C}$ below [7]. Although northern limit of distribution of $O$. smaragdina in LGM is not clear, the Bengal Delta is supposed to be out of the limit. After LGM, as the climate changed to warmer, the populations would extended to northward and come to Bengal Delta simultaneously. The populations extended to Bengal Delta were not uniform, because each of $m t \mathrm{DNA}$ types have a history of diversification in somewhere south in India and SE Asia. The diversification time was estimated in the Pliocene to Pleistocene. The recent phylogeographic studies on insects, plants, amphibians or fishes shown the significance of Pleistocene climate changes and biogeographic barriers like mountains, rivers, seas and desserts alter the diversification, radiation and isolation of new genetic lineages within many species [26]. This climatic oscillation played the major role of forming such distribution of species by changing the genetic structure and diversity [2]. The case of the distribution pattern of $O$. smaragdina in Bangladesh can be correlated with the distribution pattern of Asian elephants. Vidya et al. explained that in the case of the elephant haplotypes in Myanmar, rather suggested that these haplotypes did not arise within Myanmar, but instead resulted from a northward range expansion of beta clade haplotypes during warm period from both Sri Lanka and the Sunda region followed by subsequent admixture in this region [30].
The Bengal Basin, which is the major basin of Bengal formation [18], began 127 million years ago that after the age of glaciation, as a new vacant area with newly growing vegetation favored by the tropical climate facilitate the chance of occurrences of both the types.

The results of the study suggestseveral patterns of dispersals of both Indian and SE type of $O$. smaragdina scenario correlated with the time age of Last Glacial Maximum (LGM).

\section{Conclusions}

Oecophylla smaragdina has the evolutionary importance in Bangladesh. Based on the phylogenetic study inferred by mitochondrial $\mathrm{COI}$ and Cytb genes, it was resulted that western Bangladesh population of $O$. smaragdina is dominated by Indian type, the eastern Bangladesh population are of SE Asian types and the mixture of the two types were found in the central parts of Bangladesh that may lead to hybridized scenario. The divergence time analysis resulted that Indian type diverged ca. 2.2 Mya and SE Asian type diverged ca. 1.32 Mya corresponding to early to late Pleistocene.

\section{Acknowledgements}

We are thankful to the authority of Bangabandhu Sheikh Mujibur Rahman Agricultural University (BSMRAU), Bangladesh. We are also thankful to Dr. Akinori Ozaki, Institute of Tropical Agriculture for providing facilities during sampling in Bangladesh. We express our sincere gratitude to Dr. Masaru Matsumoto, Institute of Tropical Agriculture of Kyushu University, for his technical support. We are grateful to Dr. Noriko Azuma and Ms. Yukiko Asaka, Sapporo, Japan for their valuable advice and suggestions. We are also thankful to Dr. Akinori Ozaki, Institute of Tropical Agriculture for providing facilities during sampling in Bangladesh. This work was supported in part by JSPS KAKENHI (Grant-in-Aid for Scientific Research (B)) Grant Number 26304014, MEXT, Japan.

\section{References}

[1] Asaka, 2010. Phylogeography of the weaver ant Oecophylla smaragdina supporting southern Indian refugia hypothesis. (Thesis). Hokkaido University, Hokkaido, Japan.

[2] Avise, J. C. 2009. Phylogeography: Retrospect and prospect. 3, 15. doi: 10.1111/j.1365-2699.2008.02032.x.

[3] Azuma, N., Kikuchi, T., Ogata, K., Higashi, S. 2002. Molecular phylogeny among Local populations of weaver ant Oecophylla smaragdina. Zoological Science 19, 1321-1328. doi: $10.2108 / z s j .19 .1321$.

[4] Azuma, N., Ogata, K., Kikuchi, T., Higashi, S. 2006. Phylogeography of Asian weaver ants, Oecophylla smaragdina. Ecological Research 21, 126-136. doi: 10.1007/s11284-005-0101-6. 
[5] Blaimer, B. B., Brady, S. G., Schultz, T. R., Lloyd, M. W., Fisher, B. L., Ward, P. S. 2015. Phylogenomic methods outperform traditional multi-locus approaches in resolving deep evolutionary history: a case study of formicine ants. BMC Evolutionary Biology 15 (274), 1-14. doi: 10.1186/s12862-015-0552-5.

[6] Bowen, G. J. 2007. Palaeoclimate: When the world turned cold. Nature 445, 607-608. doi: 10.1038/445607a.

[7] Braconnot, P., Harrison S. P., Kageyama, M., Bartlein, P. J. Masson-Delmotte, V. Abe-Ouchi, A. Otto-Bliesner B., Zhao, Y. 2012. Evaluation of climate models using palaeo-climatic data. Nature Climate Change 2, 417-424.

[8] Crozier, R. H., Crozier, Y. C., 1993. The mitochondrial genome of the honeybee Apis mellifera: Complete sequence and genome organization. Genetics. 133, 97-117. doi: 10.1111/j.1365-2583.1993.tb00131.x.

[9] Crozier, R. H., Dobric, N., Imai, H. T., Graur, D., Cornuet, J. M., Taylor, R. W. 1995. Mitochondrial-DNA sequence evidence on the phylogeny of Australian jack-jumper Ants of the Myrmecia pilosula Complex. Molecular Phylogenetics and Evolution. 4, 20-30. doi: 10.1006/mpev.1995.1003.

[10] Dlussky, G. M., Wappler, T., Wedmann, S. 2008. New middle Eocene formicid species from Germany and the evolution of weaver ants. Acta Palaeontologica. Polonia 53, 615-626. doi: 10.4202/app.2008.0406.

[11] Drummond, A. J., Ho, S. Y. W., Phillips, M. J., Rambaut, A. 2006. Relaxed phylogenetics and dating with confidence. Plos Biology 4 (5), e88. doi: 10.1371/journal.pbio.004 0088.

[12] Felsenstein, J. 1981. Evolutionary trees from DNA Sequences: A maximum likelihood approach. Molecular Biology \& Evolution 17, 368-376.

[13] Goodbred, S. L., Kuehl, S. A. 2000. The significance of large sediment supply, active tectonism, and eustasy on margin sequence development: Late Quaternary stratigraphy and evolution of the Ganges-Brahmaputra delta. Sedimentary Geology 133, 227-248. doi: 10.1016/S0037-0738(00)00041-5.

[14] Katz, M. E., Miller, K. G., Wright, J. D., Wade, B. S., Browning, J. V., Cramer, B. S., Rosenthal, Y. 2008. Stepwise transition from the Eocene greenhouse to the Oligocene icehouse. Nature Geoscience. 1, 329-334. doi: 10.1038/ngeo 179 .

[15] Lokkers, C. 1986. The distribution of the weaver ant, Oecophylla smaragdina (Fabricius) (Hymenoptera: Formicidae) in northern Australia. Australian Journal of Zoology 34 (5), 683-687. doi: 10.1071/ZO9860683.

[16] Lowe, J. J. and Walker, M. J. C. 1997. Reconstructing Quaternary Environments. 2nd Edition. 1997, Harlow, England: Prentice Hall. 446.

[17] Lunt, D. H., Zhang, D. X., Szymura, J. M., Hewitt, G. M. 1996. The insect cytochrome oxidase I gene: evolutionary patterns and conserved primers for phylogenetic studies. Insect Molecular Biology 5, 153-165. doi: 10.1111/j.13652583.1996.tb00049.x.

[18] Mannan, A., 2002. Stratigraphic evolution and geochemistry of the Neogene Surma Group, Surma Basin, Sylhet, Bangladesh. Department of Geology. University of Oulu. http://herkules.oulu.fi/issn03553191/)
[19] Nascimento, F. F., Reis, M. Dos, Yang, Z. 2017. A biologist's guide to Bayesian phylogenetic analysis. Nature Ecology and Evolution. 1, 1446-1454. doi: 10.1038/s41559-017-0280-x.

[20] Nylander, J. A. A., 2004. MrModeltest v2. Program distributed by the author. Evolutionary Biology Centre Uppsala University 2, 1-2.

[21] Posada, D., Crandall, K. A. 1998. MODELTEST: Testing the model of DNA substitution. Bioinformatics. 14, 817-818. doi: 10.1093/bioinformatics/14.9.817.

[22] Rahman, M. M., Hosoishi, S., Ogata, K. 2017a. Phylogenetic position of the western Bangladesh populations of weaver ant, Oecophylla smaragdina (Fabricius) (Hymenoptera, Formicidae). Sociobiology 64, 437. doi: 10.13102/sociobiology.v64i4.1153.

[23] Rahman, M. M., Hosoishi, S., Ogata, K. 2017b. Phylogenetic analysis reveals the overlapping distribution of the Indian and southeast Asian clades of Oecophylla smaragdina (Fabricius). Journal of Faculty of. Agriculture, Kyushu University 62 (2), 429-434.

[24] Rashid, B., Sultan-Ul-Islam, Islam, B. 2015. Sub-surface geology and evolution of the Barind tract, Bangladesh. American Journal of Earth Sciences 2, 22-38.

[25] Rashid, T., Monsur, H., Suzuki, S. 2006. A review on the quaternary characteristics of Pleistocene tracts of Bangladesh. Earth Science Reports 13 (1), 1-13.

[26] Riddle, B. R., Hafner, D. J., Alexander, L. F. 2000. Phylogeography and systematics of the Peromyscus eremicus species group and the historical biogeography of North American warm regional deserts. Molecular Phylogenetics and Evolution 17, 145-160. doi: 10.1006/mpev.2000.0841.

[27] Ronquist, F., Huelsenbeck, J. P. 2003. MrBayes 3: Bayesian phylogenetic inference under mixed models. Bioinformatics. 19 (12), 1572-1574. doi: 10.1093/bioinformatics/btg180.

[28] Sameshima, S., Hasegawa, E., Kitade, O., Minaka, N., Matsumoto, T. 1999. Phylogenetic comparison of endosymbionts with their host ants based on molecular evidence. Zoological Sciences 16, 993-1000. doi: Doi 10.2108/Zsj.16.993.

[29] Tamura, K., Stecher, G., Peterson, D., Filipski, A., Kumar, S. 2013. MEGA6: Molecular evolutionary genetics analysis version 6.0. Molecular Biology and Evolution. 30, 2725-2729. doi: 10.1093/molbev/mst197.

[30] Vidya, T. N., Sukumar, R., Melnick, D. J. 2009. Range-wide mtDNA phylogeography yields insights into the origins of Asian elephants. Proceedings of Biological sciences of The Royal Society 276, 893-902276, 893-902. doi: 10.1098/rspb.2008.1494.

[31] Wetterer, J. K. 2017. Geographic distribution of the weaver ant Oecophylla smaragdina. Asian Myrmecology 9, 1-12. doi: 10.20362/am.009004.

[32] Yasmin, R., Shahid, S., Keramat, M. 2013. Evaluation of subsurface geologic formation in the Barind tract. Geotechnical and Geological Engineering 34 (5), p 1395. 